\title{
Generalização da prescrição de manejo por classe diamétrica para floresta heterogênea multiânea
}

\author{
Generalization of the diametrical class management prescription for \\ heterogeneous multiage forest
}

\author{
Daniel Dantas ${ }^{1}$ (1), Marcela de Castro Nunes Santos Terra ${ }^{1}$ (i), Natalino Calegario ${ }^{1}$ (1) \\ ${ }^{1}$ Universidade Federal de Lavras - UFLA. Lavras, MG, Brasil
}

Como citar: Dantas, D., Terra, M. C. N. S., \& Calegario, N. (2020). Generalização da prescrição de manejo por classe diamétrica para floresta heterogênea multiânea. Scientia Forestalis, 48(128), e3407.

https://doi.org/10.18671/scifor.v48n128.20

\begin{abstract}
Resumo
A prática do manejo florestal sustentável envolve muitos desafios. Uma vez que a prescrição do manejo é feita com base na modelagem da estrutura diamétrica da floresta, tem-se que quanto maior a precisão do ajuste da função à distribuição da floresta maior a precisão das prescrições do manejo. Assim, os objetivos deste trabalho foram (i) testar a performance de modelos não lineares no ajuste à distribuição diamétrica de uma floresta inequiânea; e (ii) propor uma generalização do método BDq visando uma melhor prescrição de manejo, que garanta a sustentabilidade da atividade. Foram testados 5 modelos, dentre eles o modelo de Meyer (mais comumente utilizado) e o modelo Biexponencial. A comparação dos ajustes foi realizada por meio do erro padrão residual, critério de informação de Akaike, gráficos de resíduos e teste qui-quadrado. O modelo Biexponencialfoi o de melhor desempenho, com erro relativo médio de 7,13\%, enquanto no modelo de Meyer este valor foi de 70,08\%. Verificou-se diferentes prescrições de corte pelos dois modelos, uma vez o que o modelo de Meyer permite maior exploração das maiores classes de diâmetro enquanto o Biexponencial permite maior exploração das classes iniciais. A exploração excessiva das classes de maior diâmetro pode prejudicar a produção contínua e a sustentabilidade da atividade.
\end{abstract}

Palavras-chave: Modelo biexponencial; Método BDq; Floresta nativa.

\begin{abstract}
The practice of sustainable forest management involves many challenges. Since the management prescription is based on the modeling of the forest's diameter structure, it is expected that the higher the accuracy of the function fitting, the greater the accuracy of the management prescriptions. Thus, the objectives of this work were (i) to test the performance of nonlinear models in the fitting to the diametric distribution of an unequal forest; and (ii) propose a generalization of the BDq method aiming at a better management prescription, which guarantees the sustainability of the activity. Five models were tested, among them the Meyer model (most commonly used) and the Biexponential model. The comparison of the fittings was performed using the residual standard error, Akaike information criterion, chi-squared test and scatter plots of residuals distribution. The Biexponential model presented better performance, with a mean relative error of $7.13 \%$, while the Meyer model presented a $70.08 \%$ relative error. The cut prescriptions of the two models presented differences, since the Meyer model allows greater exploration of the larger diameter classes while the Biexponential model allows for a greater exploration of the initial classes. Excessive exploitation of larger diameter classes may impair continuous production and sustainability of the activity.
\end{abstract}

Keywords: Biexponential model; BDq method; Native forest.

Fonte de financiamento: Nenhuma.

Conflito de interesse: Nada a declarar.

Autor correspondente: dantasdaniel12@yahoo.com.br

Recebido: 15 junho 2019.

Aceito: 21 novembro 2019

Editor: Paulo Henrique Müller Silva.

(c) (1) Este é um artigo publicado em acesso aberto (Open Access) sob a licença Creative Commons Attribution, que permite uso, distribuição e reprodução cc. em qualquer meio, sem restrições desde que o trabalho original seja corretamente citado. 


\section{INTRODUÇÃO}

O Brasil possui a segunda maior área florestal do mundo, abrangendo 516 milhões de hectares e, desse total, 98,7\% é composto por florestas naturais (Serviço Florestal Brasileiro, 2010), o que destaca a importância da conservação estratégica, do respeito à legislação ambiental vigente e da exploração racional dos recursos florestais como forma de alavancagem do desenvolvimento nacional. Nesse contexto, práticas de Manejo Florestal Sustentável (MFS) visam satisfazer objetivos econômicos, ecológicos e socioculturais por meio da integração de gestão, planejamento espacial e o manejo florestal criterioso (Angelstam et al., 2019).

No entanto, práticas de MFS demandam mais estudos para melhorar sua efetividade. Um aspecto crucial envolvido no MFS é a geração de prognósticos para a exploração florestal, a chamada determinação do corte, que depende de informações relacionadas a estrutura e dinâmica florestal para a efetivação de uma exploração racional de florestas heterogêneas. Estrutura e dinâmica florestal são geralmente analisadas em termos da distribuição das árvores em classes diamétricas. As distribuições diamétricas variam entre os diferentes tipos de florestas, tanto em amplitude como em forma. Por isso, a distribuição de diâmetro é característica importante do estoque em crescimento (Madi et al., 2017; Westphal et al., 2006).

Ainda dentro deste contexto, vários autores salientam que a curva de distribuição de diâmetros dos indivíduos arbóreos de uma floresta inequiânea segue uma distribuição exponencial negativa, assemelhando-se à forma de J-reverso, onde a maior quantidade de indivíduos encontra-se nas menores classes de diâmetros (e.g. Meyer, 1952; Terra et al., 2018a). De Liocourt (1898) foi o primeiro a demonstrar que a curva normal da distribuição diamétrica de uma floresta inequiânea corresponde a uma progressão geométrica decrescente. Conforme descrito por Assmann (1970): $A_{1} ; A_{q-1} ; A_{q-2} ; \ldots ; A_{q(1-n)}$, em que $A$ representa o número de árvores na menor classe de diâmetro; $q$ o quociente que determina a forma da curva e $n$ o número de classes de diâmetro.

A partir da modelagem da distribuição diamétrica é possível fazer o cálculo da estrutura balanceada e identificar classes com superávit, isto é, árvores que podem ser exploradas sem prejuízos à estrutura da floresta e sem comprometimento da produção futura (Meyer et al., 1961). Segundo Siminski et al. (2004), técnicas mais elaboradas de prescrição de corte, que incorporam além da medida de distribuição, outros parâmetros como aárea basal remanescente e diâmetro máximo desejado (O'Hara, 2014) proporcionam ganhos no sentido de garantia de sustentabilidade e adequação à legislação. Esse método é conhecido na literatura florestal como BDq (Baker et al., 1996) e comumente utiliza o modelo de Meyer (Hess et al., 2014) para a descrição da estrutura diamétrica.

Dada a heterogeneidade de condições e estruturas de florestas naturais, refinamentos podem ser aplicados a essa abordagem. Um ponto crítico nessa abordagem é a busca por modelos de melhor aderência à distribuição diamétrica da floresta. Nesse sentido, quanto maior a precisão do ajuste da função à distribuição da floresta maior a precisão das prescrições do manejo. Assim, os objetivos deste trabalho foram (i) testar a utilização de diferentes modelos de distribuição diamétrica para floresta inequiânea, visando fornecer subsídios para ações de manejo e conservação mais acuradas e (ii) avaliar a precisão das prescrições do melhor modelo ajustado em relação ao desempenho do modelo comumente utilizado (Meyer).

\section{MATERIAL E MÉTODOS}

\section{Área de estudo e coleta de dados}

O trabalho foi realizado em uma área que constitui a Reserva Florestal da Universidade Federal de Lavras, com 5,04 hectares, localizada nas coordenadas geográficas aproximadas de $21^{\circ} 1340^{\prime \prime} \mathrm{S}$ e $44^{\circ} 3750^{\prime \prime} \mathrm{W}$, a uma altitude média de $935 \mathrm{~m}$. Conforme a classificação de Köppen, o tipo climático é Cwb, com temperatura média de $19,4^{\circ} \mathrm{C}$ e precipitação anual média de $1530 \mathrm{~mm}$. Os solos são do tipo Latossolo roxo distrófico (epiálico) com textura muito 
argilosa (Junqueira-Junior et al., 2017; Terra et al., 2018b). Este fragmento de mata atlântica é classificado como floresta estacional semidecidualmontana, com fisionomia vegetacional caracterizada por até $50 \%$ de árvores caducifólias. A Reserva tem mantido suas dimensões e não sofreu corte raso desde aproximadamente 1920. Em 1987, a Reserva Florestal da UFLA sofreu demarcação de uma rede de 126 parcelas contíguas de $400 \mathrm{~m}^{2}(20 \times 20 \mathrm{~m})$, onde vem sendo realizados censos florestais, dos quais foram obtidos os dados utilizados neste trabalho, com medição dos Diâmetros à Altura do Peito (DAP) superiores a $5 \mathrm{~cm}$ e altura, totalizando 4722 indivíduos.

\section{Método usual da prescrição}

Convencionalmente, o número de árvores por classe diamétrica é estimado como uma função do valor do centro da classe, normalmente não discriminada por espécie e com um ou mais parâmetros estimados, conforme a expressão (1):

$\hat{N}_{i}=f\left(D A P_{i}, \beta\right)$

Em que $\hat{N}_{i}$ representa o número de árvores estimado por hectare, da i-th classe de DAP, e $\beta$ é o vetor de parâmetros. A função predominantemente utilizada na expressão (1) é a exponencial negativa com dois parâmetros, também conhecida como função de Meyer (1933) (2), a qual é dada por:

$N\left(D A P_{i}, \beta\right)_{i}=\beta_{0} e^{\beta_{1} D A P_{i}}+\varepsilon_{i}$

Uma variação da função (2) é a substituição do primeiro parâmetro pela exponencial do mesmo, conforme expressão a seguir:

$N\left(D A P_{i}, \beta\right)_{i}=e^{\beta_{0}} e^{\beta_{l} D A P_{i}}+\varepsilon_{i}=e^{\beta_{0}+\beta_{l} D A P_{i}}+\varepsilon_{i}$

A transformação logarítmica que ocorre na expressão (3) tem como vantagem, em relação à expressão (2), o fato de gerar uma equação linear simples, sem a necessidade da retransformação de um dos parâmetros.

Para a estimativa dos parâmetros da função (3), um novo quociente de DeLiocourt (q') é especificado, conforme expressão (4):

$q^{\prime}=\frac{E\left[N_{i}\right]}{E\left[N_{i+1}\right]}=\frac{e^{\hat{\beta}_{0}+\hat{\beta}_{1} D A P_{i}}}{e^{\hat{\beta}_{0}+\hat{\beta}_{1} D A P_{i+1}}}$

Em que $E\left(N_{i}\right)$ representa a esperança matemática do número de plantas da i-th classe diamétrica e $\hat{\beta}_{o}$. e $\hat{\beta}_{1}$ são os novos parâmetros a serem estimados para o valor especificado de $q^{\prime}$. Aplicando o logaritmo neperiano em ambos os lados da expressão (4), tem-se:

$$
\begin{aligned}
& \ln \left(q^{\prime}\right)=\ln \left(e^{\hat{\beta}_{0}^{\prime}+\hat{\beta}_{1}^{\prime} D A P_{i}}\right)-\ln \left(e^{\hat{\beta}_{0}^{\prime}+\hat{\beta}_{l}^{\prime} D A P_{i+1}}\right)=\hat{\beta}_{0}^{\prime}+\hat{\beta}_{1}{ }_{1} D A P_{i}-\hat{\beta}_{0}^{\prime}-\hat{\beta}_{l}^{\prime} D A P_{i+1} \\
& =\hat{\beta}_{1}{ }_{1} D A P_{i}-\hat{\beta}_{1}{ }_{1} D A P_{i+1}=\hat{\beta}_{l}{ }_{l}\left(D A P_{i}-D A P_{i+1}\right)
\end{aligned}
$$

Então: 


$$
\hat{\beta}_{1}^{\prime}=\frac{\ln \left(q^{\prime}\right)}{\left[\left(D A P_{i}-D A P_{i+1}\right)\right]}=\frac{\ln \left(q^{\prime}\right)}{\Delta D}
$$

Em que $\hat{\beta}_{1}^{\prime}$ é o parâmetro a ser estimado para a estruturadiamétrica proposta após a prescrição do plano de manejo e $\Delta D$ é o intervalo de classe diamétrica.

Para a obtenção do parâmetro $\hat{\beta}^{\prime}{ }_{0}$ para a estrutura proposta é necessário especificar a área basal remanescente e o diâmetro máximo que será deixado na floresta. A área basal remanescente é dada pela seguinte expressão:

$$
G_{m^{2} \cdot h a^{-l}}=\sum_{i=1}^{n} \frac{\pi}{40000} D A P_{i}^{2} \times \hat{N}_{i}=\sum_{i=1}^{n} \frac{\pi}{40000} D A P_{i}^{2} \times e^{\hat{\beta}_{\prime^{\prime}}+\hat{\beta}_{l}^{\prime} D A P_{i}}=\sum_{i=1}^{n} \frac{\pi}{40000} D A P_{i}^{2} \times e^{\hat{\beta}_{o}^{\prime}} \times e^{\hat{\beta}_{1}^{\prime} D A P_{i}}
$$

Em que $i$ representa a classe diamétrica e $n$ a classe máxima a ser deixada após a prescrição dos desbastes. Aplicando o logaritmo em ambos os lados da expressão (7), tem-se:

$$
\ln \left(G_{m^{2} \cdot h a^{-1}}\right)=\ln \left(e^{\hat{\beta}^{\prime}{ }_{0}}\right)+\ln \left(\frac{\pi}{40000} \sum_{i=1}^{n} D A P_{i}^{2} \times e^{\hat{\beta}_{1}^{\prime} D A P_{i}}\right)=\hat{\beta}_{0}{ }_{0}+\ln \left(\frac{\pi}{40000} \sum_{i=1}^{n} D A P_{i}^{2} \times e^{\hat{\beta}_{l}^{\prime} D A P_{i}}\right)
$$

Isolando o $\hat{\beta}_{0}^{\prime}$, tem-se que:

$$
\hat{\beta}_{0}^{\prime}=\ln \left(\frac{40000 \times G_{m^{2}} . h a^{-1}}{\pi \sum_{i=1}^{n} D A P_{i}^{2} \times e^{\hat{\beta}_{l}^{\prime} D A P_{i}}}\right)
$$

Portanto, o número de árvores remanescentes por classe diamétrica para a nova estrutura da floresta pode ser estimado pela seguinte expressão:

$$
\hat{N}\left(G, D A P_{\max }, q^{\prime}\right)_{i}=\underbrace{\frac{40000 \times G_{m^{2}} . h a^{-1}}{\pi \sum_{i=1}^{n} D A P_{i}^{2} \times e^{\hat{\beta}_{l}^{\prime} D A P_{i}}}}_{\beta_{0}^{\prime}} \times e^{\underbrace{\frac{\ln \left(q^{\prime}\right)}{\Delta D} \times D A P_{i}}_{\hat{\beta}_{i}^{\prime}}}
$$

Em que $G$ é a área basal remanescente por hectare, $q$ ' é o novo quociente de DeLiocourt e $n$ refere-se ao número máximo de classes diamétricas remanescentes após o desbaste proposto. A expressão (10) pode ser utilizada para estimativa por espécie, grupo de espécies ou para toda a floresta.

\section{Generalização do método}

Em tese, a função dada pela expressão (1) não necessariamente deve ser a função de Meyer (2). Qualquer função que se ajuste à forma exponencial negativa pode ser utilizada para o propósito de manejo. Como candidatas, existem várias funções na literatura (Mervart, 1972; Ratkowsky; Giles, 1990; Pinheiro; Bates, 2000) que se aproximam de tal forma e que, neste caso, podem substituir a função de Meyer e, provavelmente, gerar ajustes mais precisos. Quanto maior a precisão do ajuste da função à distribuição da floresta maior a precisão das prescrições do manejo.

Algumas destas funções estão representadas em (11), sendo as expressões 11.a (Mervart, 1972) e 11.b (Ratkowsky \& Giles,1990), como Meyer, são funções de dois parâmetros. A expressão 11.c (Ratkowsky \& Giles, 1990) é representada por três parâmetros, enquanto a expressão 11.d (Pinheiro \& Bates, 2000) possui quatro parâmetros. 


$$
\begin{aligned}
& n_{i}(d, \alpha, \beta)=\alpha d_{i}^{\beta}+\varepsilon_{i} \rightarrow \text { para } \beta<0 \\
& n_{i}(d, \alpha, \beta)=\alpha\left(1+d_{i}\right)^{\beta}+\varepsilon_{i} \rightarrow \text { para } \beta<0 \\
& n_{i}(d, \alpha, \beta, \lambda)=\alpha \times \exp \left(\frac{\beta}{d_{i}+\lambda}\right)+\varepsilon_{i} \rightarrow \text { para } \beta>0 \\
& n_{i}(d, \alpha, \beta, \lambda, \varphi)=\alpha \times \exp \left(-\exp (\beta) d_{i}\right)+\lambda \times \exp \left(-\exp (\varphi) d_{i}\right)+\varepsilon_{i} \rightarrow \text { para }, \lambda>0 e \beta, \varphi<0
\end{aligned}
$$

\section{Geração da nova estrutura com base na estimativa de novos parâmetros}

Como no caso de Meyer, as funções acima podem ser utilizadas para a representação da estrutura original e de uma nova estrutura da floresta após certa intervenção proposta por um plano de manejo. Para a função (11.a), por exemplo, as estimativas dos novos parâmetros podem ser obtidas conforme as seguintes etapas:

a) Define-se um novo valor para q;

$$
q^{\prime}=\frac{N_{i}}{N_{i+1}}=\frac{\hat{\alpha}^{\prime} D A P_{i}^{\hat{\beta}^{\prime}}}{\hat{\alpha}^{\prime} D A P_{i+1}^{\hat{\beta}^{\prime}}}
$$

b) Aplicando o logaritmo em ambos os lados e isolando-se o $\hat{\beta}^{\prime}$, tem-se que:

$$
\hat{\beta}^{\prime}=\frac{\ln \left(q^{\prime}\right)}{\ln \left(D A P_{i}\right)-\ln (D A P \mid i+l)}
$$

c) Para a estimativa do segundo parâmetro, pode-se usar o mesmo procedimento no caso da função de Meyer, ou seja, limita-se a área basal e isola-se o parâmetro, conforme expressão a seguir:

$$
G_{m^{2} \cdot h a^{-l}}=\sum_{i=1}^{n} \frac{\pi}{40000} D A P_{i}^{2} \times \hat{N}_{i}=\sum_{i=1}^{n} \frac{\pi}{40000} D A P_{i}^{2} \times \hat{\alpha}^{\prime} \times D A P_{i}^{\hat{\beta}^{\prime}}
$$

Isolando o parâmetro $\hat{\alpha}^{\prime}$ da expressão (14), tem-se:

$$
\begin{aligned}
& \hat{\alpha}^{\prime}=\frac{G_{m^{2} \cdot h a^{-1}}}{\frac{\pi}{40000} \sum_{i=1}^{n} D A P_{i}^{\left(2+\hat{\beta}^{\prime}\right)}} \\
& \hat{N}\left(G, D A P_{\max }, q\right)=\underbrace{\frac{G_{m^{2} \cdot h a^{-1}}}{\frac{\pi}{40000} \sum_{i=1}^{n} D A P_{i}^{\left(2+\hat{\beta}^{\prime}\right)}}}_{\hat{\alpha}^{\prime}} \times D A P_{i} \underbrace{\frac{\ln \left(q^{\prime}\right)}{\ln \left(D A P_{i}\right)-\ln (D A P \mid i+1)}}_{\hat{\beta}^{\prime}}
\end{aligned}
$$

O mesmo procedimento pode ser adotado para a Equação 11.b, devido ao fato de ambas possuírem dois parâmetros. Como são duas equações, uma do novo $q$ e outra para a área basal remanescente, e dois parâmetros, o sistema é possível de ser resolvido. Para as 
equações de mais de dois parâmetros a generalização do método será baseada na estimativa da nova estrutura como uma função direta da área basal remanescente e do novo valor de $q$.

\section{Geração da nova estrutura com base na área basal remanescente e no novo valor de $\boldsymbol{q}$}

Para funções com mais de dois parâmetros, uma alternativa de se estimar a nova estrutura, após a proposta de manejo, é dada pelas seguintes etapas:

a) Definir novos valores da razão do número de indivíduos entre classes diamétricas (q'), da área basal remanescente $\left(G^{\prime}\right)$ e da classe diamétrica máxima $\left(D_{\text {max }}\right)$;

b) Estimar a nova área basal para a primeira classe diamétrica $\left(G_{1}{ }^{\prime}\right)$ tomando como base a mesma redução proporcional da área basal total;

c) Estimar o número de árvores para a primeira classe com base na nova área basal $\left(\mathrm{G}_{1}{ }^{\prime}\right)$;

d) Estimar o número de árvores das demais classes com base no novo valor de q', até o $D_{\text {maxi }}$

e) Redistribuir a diferença entre a área basal remanescente e a área basal remanescente desejada entre as classes diamétricas com base na proporção entre o número de indivíduos da classe e o número total de indivíduos.

Após as etapas acima, uma nova estrutura é gerada sendo possível a estimativa dos parâmetros para qualquer função, independentemente do número de parâmetros, utilizando um método não linear iterativo.

As funções propostas, representadas em (11), foram ajustadas utilizando o método não linear de Gauss Newton. Como em qualquer outro estudo de modelagem, a função a ser selecionada será aquela que for mais precisa em representar à base de dados, nesse sentido os critérios de avaliação dos modelos foram a significância dos parâmetros pelo teste $t$ student, a um nível de 0,05, o erro padrão residual (Syx) (17), gráficos de dispersão dos resíduos, Coeficiente de determinação ajustado $\left(\bar{R}^{2}\right)(18)$, Critério de Informação de Akaike (Sakamoto et al., 1986) (19), em que menores valores indicam os melhores modelos, e o teste qui-quadrado $\left(X^{2}\right)$ de Pearson.

$$
\begin{aligned}
& \operatorname{Syx}(\%)=\frac{\sqrt{\frac{S Q R}{n-p-1}}}{\bar{Y}} * 100 \\
& \bar{R}^{2}=1-\frac{n-1}{n-p} * \frac{S Q R}{S Q T}
\end{aligned}
$$

em que: $n$ é o número de casos; $p$ é o número de parâmetros; $\bar{Y}$ é a média dos valores observados; SQR é a soma de quadrados do resíduos; e SQT é a soma de quadrados total.

$$
A I C=-2 * \ln (m v)+2 p
$$

em que: $A / C$ é o Critério de Informação de Akaike; In é o logaritmo neperiano; $m v$ = valor de máxima verossimilhança; $p$ é o número de parâmetros do modelo.

\section{RESULTADOS E DISCUSSÃO}

Os indivíduos estão distribuídos em classes de diâmetro com intervalos de $6 \mathrm{~cm}$ e valores centrais variando de 7 a $61 \mathrm{~cm}$. Os parâmetros ajustados para as funções propostas e seus respectivos Erros Padrão Residuais (EPR) são apresentados na Tabela 1. Todos os parâmetros foram significativos a um nível de 0,05, pelo teste t-Student. 
Tabela 1. Parâmetros ajustados para os modelos propostos e seus respectivos erro padrão residuais (EPR), Critérios de Informação de Akaike (AIC) e $R^{2}$ ajustado $\left(R^{2}\right)$.

\begin{tabular}{cccccccc}
\hline MODELO & $\hat{\alpha}$ & $\hat{\beta}$ & $\hat{\lambda}$ & $\hat{\varphi}$ & EPR & AIC & $\overline{\mathbf{R}}^{2}$ \\
\hline a & 374130,7866 & $-2,4395$ & - & - & 25,93 & 97,25 & 0,9994 \\
b & 848045,3833 & $-2,6766$ & - & - & 29,89 & 100,09 & 0,9992 \\
c & 7,9392 & 105,1210 & 10,4785 & - & 22,07 & 94,70 & 0,9996 \\
d & 28275,3477 & $-1,1108$ & 633,9595 & $-2,8842$ & 17,52 & 90,54 & 0,9998 \\
Meyer & 17392,6669 & $-0,2401$ & - & - & 81,52 & 120,16 & 0,9945 \\
\hline
\end{tabular}

Como pode ser visto na Tabela 1, as equações com três e quatro parâmetros, 11.c e 11.d,respectivamente, resultaram em menores valores de EPR. Esta superioridade pode ser atribuída ao fato de que o parâmetro $\lambda$ está relacionado com a taxa média em que ocorre o crescimento/decrescimento da curva, e o parâmetro $\varphi$ proporciona um aumento na flexibilidade do modelo no ajuste dos dados (Sheiner \& Beal, 1981). Assim, a função Biexponencial teve a melhor performance dentre as funções testadas, com um erro padrão residual de 17,52, valor este quatro vezes menor do que o erro da função de Meyer (70,08\%), utilizada como referência neste estudo.

Os dados utilizados apresentaram uma distribuição do tipo J-reverso, comumente observada em florestas naturais, padrão que deve ser mantido mesmo após a realização de intervenções silviculturais (Podlaski \& Zasada, 2008; Rubin et al., 2006; Westphal et al., 2006; Zheng \& Zhou, 2010). A distribuição J-reverso descreve a maior concentração de indivíduos nas menores classes de diâmetro, portanto é um indicador do potencial sustentável da floresta baseado na estrutura, uma vez que sinaliza o suprimento das maiores classes de diâmetro à medida em que as árvores crescem na floresta. Ecologicamente, a manutenção dessa curva é explicada pelas taxas de mortalidade iguais entre as classes de diâmetro (Westphal et al., 2006).

A Figura 1 demonstra que à medida que o diâmetro aumenta, a frequência dos indivíduos decresce exponencialmente à direita e as funções de Meyer e Biexponencial conseguiram captar o deslocamento desta distribuição. A função Biexponencial foi capaz de explicar melhor a variabilidade dos dados, quando comparada com a função de Meyer, uma vez que existe menor distância dos valores estimados por aquela função em relação aos valores observados, principalmente para as maiores classes diamétricas. Essas menores distâncias refletem os menores erros.

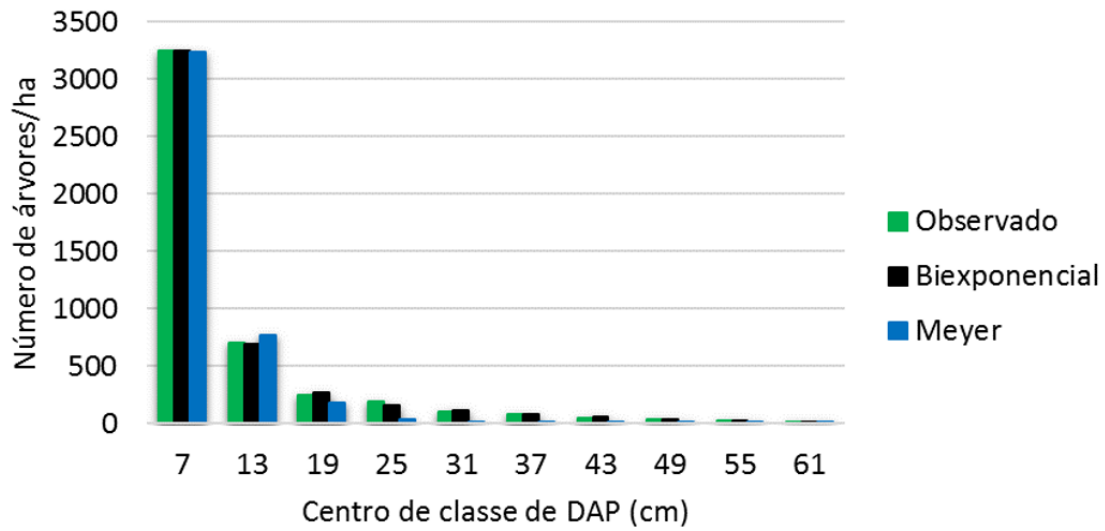

Figura 1. Distribuição observada e estimada dos indivíduos arbóreos em classes de diâmetro.

Os gráficos de resíduos (Figura 2) indicam superioridade do modelo Biexponencial (11.d) na modelagem do número de indivíduos por classe de diâmetro, em relação aos demais modelos analisados. Verifica-se distribuição mais homogênea e baixa dispersão de resíduos, com valores compreendidos no intervalo de -17 a 17\%. Enquanto o modelo de Meyer resultou em uma maior dispersão de resíduos, com valores entre -9 e 99\%, indicando uma tendência de subestimar o número de indivíduos, especialmente nas maiores classes de diâmetros. $\mathrm{O}$ erro relativo médio para 
o modelo Biexponencial foi de 7,13\%, enquanto para o modelo de Meyer o valor foi 70,08\%. Sendo o ERM máximo para o Biexponencial de 17,05\%, e para Meyer, 99,96\%.
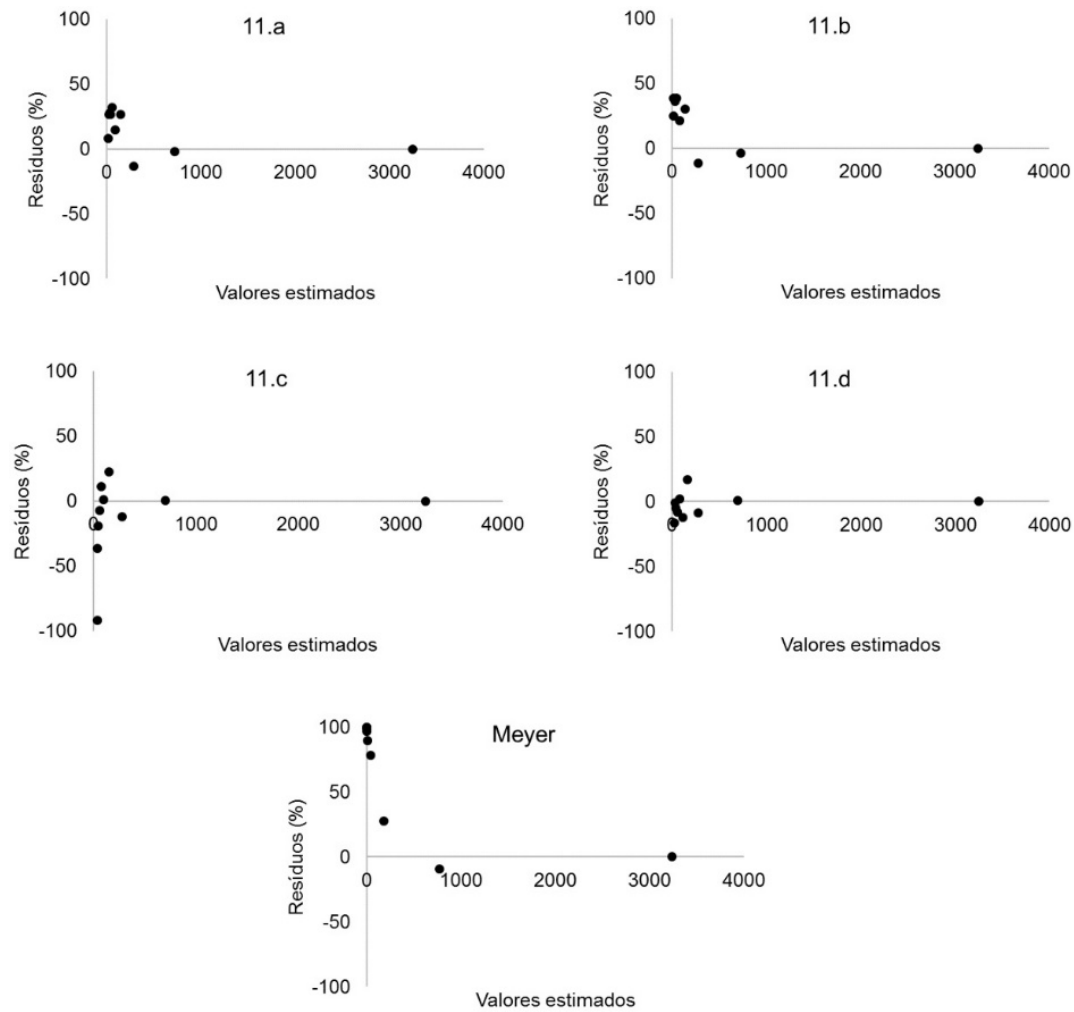

Figura 2. Gráficos de distribuição de resíduos.

Os valores estimados por classe diamétrica pela função de Meyer e os valores estimados pela função Biexponencial foram comparados por meio do teste qui-quadrado, que resultou em um valor-p inferior a 0,05 ( $p=7,49 \mathrm{E}-90)$, com isso a hipótese nula foi rejeitada indicando diferença significativa entre as estimativas. Verifica-se que a função de Meyer apresentou forte tendência em subestimar o número de plantas nas classes diamétricas com centros acima de $19 \mathrm{~cm}$ (Tabela 2), enquanto a função Biexponencial conseguiu representar de forma mais adequada a estrutura original da floresta.

Tabela 2. Números de árvores observados $(\mathrm{N})$ e estimados por classe de diâmetro (d), pelos modelos de Meyer e Biexponencial, com os respectivos Erros Relativos Médios (ERM) e valores do quociente de DeLiocourt.

\begin{tabular}{|c|c|c|c|c|c|c|c|}
\hline \multirow{2}{*}{$\begin{array}{c}\text { Centro } \\
\text { de } \\
\text { classe }\end{array}$} & \multirow{2}{*}{$\mathbf{N}$} & \multirow{2}{*}{ Biexponencial } & \multirow{2}{*}{ Meyer } & \multirow{2}{*}{$\begin{array}{c}\text { ERM (\%) } \\
\text { Biexp }\end{array}$} & \multirow{2}{*}{$\begin{array}{c}\text { ERM (\%) } \\
\text { Meyer }\end{array}$} & \multirow{2}{*}{ q' Biexp } & \multirow{2}{*}{ q' Meyer } \\
\hline & & & & & & & \\
\hline 7 & 3249 & 3249,26 & 3239,84 & 0,01 & 0,28 & - & - \\
\hline 13 & 702 & 697,61 & 767,26 & 0,63 & 9,3 & 4,66 & 4,22 \\
\hline 19 & 251 & 273,4 & 181,7 & 8,92 & 27,61 & 2,55 & 4,22 \\
\hline 25 & 198 & 164,23 & 43,03 & 17,05 & 78,27 & 1,66 & 4,22 \\
\hline 31 & 101 & 113,1 & 10,19 & 11,98 & 89,91 & 1,45 & 4,22 \\
\hline 37 & 82 & 80,27 & 2,41 & 2,11 & 97,06 & 1,41 & 4,22 \\
\hline 43 & 53 & 57,31 & 0,57 & 8,14 & 98,92 & 1,4 & 4,22 \\
\hline 49 & 39 & 40,97 & 0,14 & 5,05 & 99,65 & 1,4 & 4,22 \\
\hline 55 & 29 & 29,29 & 0,03 & 1,01 & 99,89 & 1,4 & 4,22 \\
\hline 61 & 18 & 20,95 & 0,01 & 16,37 & 99,96 & 1,4 & 4,22 \\
\hline
\end{tabular}


No modelo de Meyer, a taxa de decrescimento do número de indivíduos é constante, ou seja, permanece a mesma independentemente da classe diamétrica. Já o modelo Biexponencial é a soma de duas curvas exponenciais com diferentes taxas de decrescimento. Os parâmetros $\alpha$ e $\beta$ representam a taxa de decrescimento rápido enquanto os parâmetros $\lambda$ e $\varphi$ representam a taxa de decrescimento lento. Sendo assim, quando a floresta apresenta uma distribuição irregular ou não equilibrada, em que a razão entre as classes diamétricas não é constante, o modelo Biexponencial tem uma maior sensibilidade para captar essas diferenças na taxa de redução do número de indivíduos ao longo da curva, como ocorreu no presente estudo.

Como o objetivo da utilização destas funções é representar uma nova estrutura da floresta após certa intervenção proposta por um plano de manejo, quanto mais precisas forem as estimativas geradas, mais eficiente e sustentável será o manejo. Nesse sentido, considerando o melhor desempenho da função Biexponencial, alternativas de manejo baseadas nos valores estimados por esta função podem proporcionar uma maior precisão na quantificação do estoque de madeira existente do que a função de Meyer.

A generalização do método $\mathrm{BDq}$ foi aplicada utilizando o modelo de Meyer e o Biexponencial, para um diâmetro máximo remanescente de $43 \mathrm{~cm}$ e uma área basal remanescente de $60 \%\left(49,38 \mathrm{~m}^{2}\right)$ da área basal original.

Os coeficientes da função de Meyer foram recalculados e pode-se, assim, definir a existência de classes superavitárias (árvores a remover) e deficitárias, as quais devem ter sua densidade aumentada, obtendo-se, assim, o número de árvores e a área basal a serem removidas de acordo com a distribuição dos indivíduos em cada classe diamétrica.

Segundo as estimativas do modelo de Meyer, há um déficit de árvores na floresta na segunda e na terceira classe (Tabela 3). Nas demais é possível a exploração de 586 árvores $\left(49,38 \mathrm{~m}^{2}\right)$. Já segundo o modelo Biexponencial, é possível a exploração de 1093 árvores $\left(49,38 \mathrm{~m}^{2}\right)$ (Tabela 4), ou seja, uma quantidade maior de árvores, porém a mesma área basal que o Meyer.

Tabela 3. Números de árvores observados, área basal, valores remanescentes e estimativas de colheitas por classe de diâmetro considerando um diâmetro máximo de $43 \mathrm{~cm}$ e área basal remanescente de 49,38 $\mathrm{m}^{2}$, pelo modelo de Meyer.

\begin{tabular}{|c|c|c|c|c|c|c|}
\hline \multirow{2}{*}{$\begin{array}{l}\text { Centro } \\
\text { de } \\
\text { Classe }\end{array}$} & \multicolumn{2}{|c|}{ Valores Observados } & \multicolumn{2}{|c|}{$\begin{array}{c}\text { Valores } \\
\text { Remanescentes }\end{array}$} & \multicolumn{2}{|c|}{ Estimativas de colheitas } \\
\hline & $\mathbf{N}$ & $\mathbf{m}^{2}$ & $\mathbf{n}$ & $\mathbf{m}^{2}$ & $\mathbf{n}$ & $\mathbf{m}^{2}$ \\
\hline 7 & 3249 & 12,5 & 2975 & 11,45 & 274 & 1,05 \\
\hline 13 & 702 & 9,32 & 1063 & 14,1 & -361 & $-4,79$ \\
\hline 19 & 251 & 7,12 & 380 & 10,76 & -129 & $-3,64$ \\
\hline 25 & 198 & 9,72 & 136 & 6,65 & 62 & 3,07 \\
\hline 31 & 101 & 7,62 & 48 & 3,65 & 53 & 3,97 \\
\hline 37 & 82 & 8,82 & 17 & 1,86 & 65 & 6,96 \\
\hline 43 & 53 & 7,7 & 6 & 0,9 & 47 & 6,8 \\
\hline 49 & 39 & 7,35 & 0 & 0 & 39 & 7,35 \\
\hline 55 & 29 & 6,89 & 0 & 0 & 29 & 6,89 \\
\hline 61 & 18 & 5,26 & 0 & 0 & 18 & 5,26 \\
\hline Total & 4722 & 82,3 & 4625 & 49,38 & 97 & 32,92 \\
\hline
\end{tabular}


Tabela 4. Números de árvores observados, área basal, valores remanescentes e estimativas de colheitas por classe de diâmetro considerando um diâmetro máximo de $43 \mathrm{~cm}$ e área basal remanescente de 49,38 $\mathrm{m}^{2}$, pelo modelo Biexponencial.

\begin{tabular}{|c|c|c|c|c|c|c|}
\hline \multirow{2}{*}{$\begin{array}{l}\text { Centro } \\
\text { de } \\
\text { Classe }\end{array}$} & \multicolumn{2}{|c|}{ Valores Observados } & \multicolumn{2}{|c|}{$\begin{array}{c}\text { Valores } \\
\text { Remanescentes }\end{array}$} & \multicolumn{2}{|c|}{ Estimativas de colheitas } \\
\hline & $\mathbf{N}$ & $\mathbf{m}^{2}$ & $\mathbf{n}$ & $\mathbf{m}^{2}$ & $\mathbf{n}$ & $\mathrm{m}^{2}$ \\
\hline 7 & 3249 & 12,5 & 2544 & 9,79 & 705 & 2,71 \\
\hline 13 & 702 & 9,32 & 546 & 7,25 & 156 & 2,07 \\
\hline 19 & 251 & 7,12 & 214 & 6,07 & 37 & 1,05 \\
\hline 25 & 198 & 9,72 & 129 & 6,31 & 69 & 3,41 \\
\hline 31 & 101 & 7,62 & 89 & 6,68 & 12 & 0,94 \\
\hline 37 & 82 & 8,82 & 63 & 6,76 & 19 & 2,06 \\
\hline 43 & 53 & 7,7 & 45 & 6,52 & 8 & 1,18 \\
\hline 49 & 39 & 7,35 & 0 & 0 & 39 & 7,35 \\
\hline 55 & 29 & 6,89 & 0 & 0 & 29 & 6,89 \\
\hline 61 & 18 & 5,26 & 0 & 0 & 18 & 5,26 \\
\hline Total & 4722 & 82,3 & 3629 & 49,38 & 1093 & 32,92 \\
\hline
\end{tabular}

Nota-se, portanto, que o modelo de Meyer é mais permissivo nas maiores classes, promovendo uma intensa exploração nas classes imediatamente anteriores ao diâmetro máximo. Nas classes de 37 e $43 \mathrm{~cm}$, por exemplo, a prescrição de manejo baseada no modelo de Meyer resultou em apenas 17 e 6 árvores remanescentes, respectivamente. Já com base no modelo Biexponencial os valores remanescentes foram 63 e 45 árvores para as mesmas classes, respectivamente. Economicamente, há um forte interesse em uma exploração mais intensa das árvores pertencentes às maiores classes diamétricas, no entanto, é preciso cautela quanto as implicações disso para os ciclos de corte futuros.

Portanto, há no modelo de Meyer, comumente utilizado na elaboração de planos de manejo, a tendência de promover a realização de um corte mais pesado nas maiores classes de diâmetro. Trabalhos apontam para a insustentabilidade de planos de manejo que promovem grande remoção de árvores de maior diâmetro. Por exemplo, Reis et al. (2010) avaliando a Floresta Nacional do Tapajós após 28 anos da exploração, verificou que $96,7 \%$ dos indivíduos possuem diâmetro inferior a $45 \mathrm{~cm}$, o que antes da exploração foi praticamente $20 \%$ inferior, demonstrando que a extração foi muito pesada. Braz (2010) salienta que a colheita das árvores nas classes de maior diâmetro reduz drasticamente a capacidade de recuperação da floresta, apontando a necessidade de longos períodos para tal recuperação.

Assim, quando se utiliza um modelo muito permissivo ao corte das árvores nas maiores classes de diâmetro, como é o modelo de Meyer, há um comprometimento da capacidade futura da floresta, ferindo os princípios da sustentabilidade. Fearnside (2008) questiona a sustentabilidade dos planos de manejo atuais, afirmando que, no primeiro ciclo, um grande número de árvores que vêm crescendo há anos são cortadas. Essa exploração, no entanto, é muito mais lucrativa no primeiro ciclo do que nos ciclos subsequentes, quando a colheita atinge um equilíbrio e o manejador só colhe o que cresceu no intervalo entre colheitas. Isso representa um risco de o manejador abandonar o sistema e recorrer à ilegalidade da colheita predatória. Nesse sentido, Reis et al. (2010) salientam que a exploração madeireira realizada sem critérios ecológicos poderá ser um risco para a biodiversidade existente, pois o comportamento das espécies remanescentes após a colheita florestal é diferente se comparada à floresta primária: a colheita pode favorecer certos grupos de espécies remanescentes e prejudicar o crescimento e estabelecimento de outras, o que pode exigir longos períodos de dinâmicas sucessionais para algumas espécies se restabelecerem na área. Ainda sobre a sustentabilidade vale ressaltar que, do ponto de vista genético, ocorre redução drástica no tamanho das populações, através do corte seletivo, podendo levar à deriva genética (Sebbenn et al., 2008; Dal Bem et al., 2015). 
Obviamente, a maior ou menor exploração das maiores classes tem implicação direta na destinação da madeira e consequentemente impacto no lucro advindo do manejo florestal. Produtos madeireiros que exigem toras mais grossas, como laminados, tem maior valor agregado (Stringer, 2014). Porém essa produção pode não ser contínua se não respeitar a estrutura e dinâmica de crescimento naturais da florestal. Portanto, um modelo que gere uma prescrição mais adequada é fundamental para garantir a sustentabilidade do manejo. Nesse sentido, reforça-se que a determinação do corte a partir do modelo Biexponencial, embora garanta menos árvores grossas no primeiro ciclo, pode colaborar para a sustentabilidade do manejo em florestas naturais, pois adequa-se melhor a estrutura diamétrica da floresta.

Assim, uma exploração com base na sustentabilidade permitirá um melhor aproveitamento do recurso madeireiro hoje e no futuro ao mesmo tempo em que garante a proteção da biodiversidade (Garrido-Filha, 2002). A exploração sustentável é feita mediante aprovação do plano de manejo, por isso, garantir a legalidade e a produção sustentável são caminhos inevitáveis para a atividade gerar ganhos duradouros e promover o desenvolvimento com benefícios sociais, sem estragos ambientais.

\section{CONCLUSÃO}

A função Biexponencial ajustou-se de forma eficiente à distribuição diamétrica exponencial negativa ("J-reverso") da floresta inequiânea estudada, com frequências estimadas mais próximas das observadas e menor erro padrão residual, comparada ao modelo de Meyer. Sendo assim, a função Biexponencial pode ser utilizada para melhorar tanto a descrição da estrutura diamétrica da floresta como as prescrições de manejo, uma vez que alternativas de manejo baseadas neste modelo possuem maior compromisso com a sustentabilidade. Mais estudos sobre aspectos do plano de manejo florestal sustentável, como diâmetro mínimo de corte, ciclos de corte, e critérios específicos para diferentes espécies são necessários para que a sustentabilidade do manejo seja garantida.

\section{AGRADECIMENTOS}

Os autores agradecem ao Programa de Pós-Graduação em Engenharia Florestal da Universidade Federal de Lavras (PPGEF-UFLA), à Coordenação de Aperfeiçoamento de Pessoal de Nível Superior (CAPES) e ao Conselho Nacional de Desenvolvimento Científico e Tecnológico pelo apoio financeiro na realização deste estudo.

\section{REFERÊNCIAS}

Angelstam, P., Elbakidze, M., Axelsson, R., Khoroshev, A., Pedroli, B., Tysiachniouk, M., \& Zabubenin, E. (2019). Model forests in Russia as landscape approach: demonstration projects or initiatives for learning towards sustainable forest management. Forest Policy and Economics, 101, 96-110. http://dx.doi.org/10.1016/j.forpol.2019.01.005.

Assmann, E. (1970). The principles of forest yield study: Studies in the organic production, structure, increment, and yield of forest stands (520 p.). Oxford: Pergamon Press.

Baker, J. B., Cain, M. D., Guldin, J. M., Murphy, P. A., \& Shelton, M. G. (1996). Uneven-aged silviculture for the loblolly and shortleaf pine forest cover types (65 p.). New Orleans, LA: U.S. Dept of Agriculture, Forest Service, Southern Forest Experiment Station. http://dx.doi.org/10.2737/SO-GTR-118.

Braz, E. M. (2010). Subsídios para o planejamento do manejo de florestas tropicais da Amazônia (Tese de doutorado). Universidade Federal de Santa Maria, Santa Maria.

Dal Bem, E. A., Bittencourt, J. V. M., De Moraes, M. L. T., \& Sebbenn, A. M. (2015). Cenários de corte seletivo de árvores na diversidade genética e área basal de populações de Araucariaangustifolia com base em modelagem Ecogene. Scientia Forestalis, 43(106), 453-466.

De Liocourt, F. (1898). De l'amenagementdessapinières (Bulletin trimestriel, pp. 396-409). Besançon: Société forestière de Franche-Comté et Belfort.

Fearnside, P. M. (2008). Amazon forest maintenance as a source of environmental services. Anais da Academia Brasileira de Ciências, 80(1), 101-114. PMid:18345379. http://dx.doi.org/10.1590/S000137652008000100006. 
Garrido-Filha, I. (2002). Manejo florestal: questões econômico-financeiras e ambientais. Estudos Avançados, 16(45), 91-106. http://dx.doi.org/10.1590/S0103-40142002000200007.

Hess, A. F., Minatti, M., Ferrari, L., \& Pintro, B. A. (2014). Manejo de floresta ombrófila mista pelo método de liocourt, município de Painel, SC. Cerne, 20(4), 575-580. http://dx.doi.org/10.1590/01047760201420041230.

Junqueira Junior, J. A., Mello, C. R., Owens, P. R., Mello, J. M., Curi, N., \& Alves, G. J. (2017). Time-stability of soil water content (SWC) in an Atlantic Forest - Latosol site. Geoderma, 288, 64-78. http://dx.doi.org/10.1016/j.geoderma.2016.10.034.

Madi, P. S., Vendruscolo, J. D. G. S., Silva, C. A., \& Lima, M. P. (2017). Univariate models to represent the diametric distribution of thinned stand of Tectonagrandis Linn. F. Advances in Forestry Science, 4(2), 119-123.

Mervart, J. (1972). 0. Frequency curves of the growing stock in the Nigerian high forest. Nigerian Journal of Forestry, 2(1), 7-15.

Meyer, H. A. (1952). Structure, growth, and drain in balanced uneven-aged forests. Journal of Forestry, 50(2), 85-92.

Meyer, H. A., Recknagel, A. B., \& Stevenson, D. D. (1961). Forest management (282 p.). New York: The Ronald Press.

Meyer, W. H. (1933). Approach of abnormally stocked forest stands of Douglas fir to normal condition. Journal of Forestry, 31, 400-406.

O'Hara, K. L. (2014). Multiaged silviculture: managing for complex forest stand structures (232 p.). Oxford: Oxford University Press. http://dx.doi.org/10.1093/acprof:oso/9780198703068.001.0001.

Pinheiro, J. C., \& Bates, D. M. (2000). Mixed-efects models in S and S-PLUS (528 p.). New York: SpringerVerlag. http://dx.doi.org/10.1007/978-1-4419-0318-1.

Podlaski, R., \& Zasada, M. (2008). Comparison of selected statistical distributions for modelling the diameter distributions in near-natural Abies-Fagus forests in the Świetokrzyski National Park (Poland). European Journal of Forest Research, 127(6), 455-463. http://dx.doi.org/10.1007/s10342-0080229-3.

Ratkowsky, D. A., \& Giles, D. E. (1990). Handbook of nonlinear regression models (241p.). New York: Marcel Dekker.

Reis, L. P., Ruschel, A. R., Coelho, A. A., Luz, A. S., \& Martins-da-Silva, R. C. V. (2010). Avaliação do potencial madeireiro na Floresta Nacional do Tapajós após 28 anos da exploração florestal. Pesquisa Florestal Brasileira, 30(64), 265-281. http://dx.doi.org/10.4336/2010.pfb.30.64.265.

Rubin, B. D., Manion, P. D., \& Faber-Langendoen, D. (2006). Diameter distributions and structural sustainability in forests. Forest Ecology and Management, 222(1-3), 427-438. http://dx.doi.org/10.1016/j.foreco.2005.10.049.

Sakamoto, Y., Ishiguro, M., \& Kitagawa, G. (1986). Akaike information criterion statistics (290 p.). Dordrecht: The Netherlands.

Sebbenn, A. M., Degen, B., Azevedo, V. C. R., Silva, M. B., de Lacerda, A. E. B., Ciampi, A. Y., Kanashiro, M., Carneiro, F. S., Thompson, I., \& Loveless, M. D. (2008). Modelling the long-term impacts of selective logging on genetic diversity and demographic structure of four tropical tree species in the Amazon forest. Forest Ecology and Management, 254(2), 335-349. http://dx.doi.org/10.1016/j.foreco.2007.08.009.

Serviço Florestal Brasileiro - SFB. (2010). Florestas do Brasil em resumo - 2010: dados de 2005-2010 (152 p.). Brasília: Serviço Florestal Brasileiro.

Sheiner, L. B., \& Beal, S. L. (1981). Evaluation of methods for estimating population pharmacokinetic parameters. II. Biexponential model and experimental pharmacokinetic data. Journal of Pharmacokinetics and Biopharmaceutics, 9(5), 635-651. PMid:7334463. http://dx.doi.org/10.1007/BF01061030.

Siminski, A., Mantovani, M., Reis, M. S., \& Fantini, A. C. (2004). Sucessão florestal secundária no município de São Pedro de Alcântara, litoral de Santa Catarina: estrutura e diversidade. Ciência Florestal, 14(1), 21-33. http://dx.doi.org/10.5902/198050981778.

Stringer, J. (2014). Hardwood times product types and specifications. Kentucky Woodlands Magazine, 9, 6-9.

Terra, M. C. N. S., Mello, C. R., Mello, J. M., Oliveira, V. A., Nunes, M. H., Silva, V. O., Rodrigues, A. F., \& Alves, G. J. (2018a). Stemflow in a neotropical forest remnant: vegetative determinants, spatial distribution and correlation with soil moisture. Trees - Structure and Functions, 32(1), 323-335. 
Terra, M. C. N. S., Mello, J. M., Mello, C. R., Santos, R. M., Coelho, P. A., Pereira, D. G. S., \& Silva, T. M. C. (2018b). Short-term changes in an ombrophilousatlantic forest. Nativa (Sinop), 6(5), 543-550. http://dx.doi.org/10.31413/nativa.v6i5.5426.

Westphal, C., Tremer, N., Oheimb, G. V., Hansen, J., Gadow, K. V., \& Härdtle, W. (2006). Is the reverse Jshaped diameter distribution universally applicable in European virgin beech forests? Forest Ecology and Management, 223(1-3), 75-83. http://dx.doi.org/10.1016/j.foreco.2005.10.057.

Zheng, L. F., \& Zhou, X. N. (2010). Diameter distribution of trees in natural stands managed on polycyclic cutting system. Forestry Studies in China, 12(1), 21-25. http://dx.doi.org/10.1007/s11632-010-0009-2.

Contribuição dos autores: DD: escrita - primeira redação, escrita - revisão e edição, investigação, conceituação; MCNST: escrita - primeira redação, escrita - revisão e edição, investigação, conceituação; NC: escrita - primeira redação, escrita - revisão e edição, metodologia, supervisão, conceituação, análise formal. 